\title{
Pursuit-locked apparent motion
}

\author{
JOYCE E. FARRELL, TERESA PUTNAM, and ROGER N. SHEPARD \\ Stanford University, Stanford, California
}

\begin{abstract}
A vertical line, illuminated in $\mathbf{n}$ brief pulses at each position, was repositioned a distance $\mathbf{D}$ to the right every $\mathrm{T}$ msec. Under some conditions, the appearance of a single line jumping from one position to the next (standard apparent motion) gave way to the striking appearance of a bundle of $n$ parallel vertical lines moving with constant velocity across the entire screen. This appearance implies the emergence of continuous pursuit eye movements, which have previously been reported for similarly discontinuous displays. Such "pursuit-locked" apparent motion spontaneously occurred when $D$ was less than about 3 deg of visual angle, and when $T$ was less than a visual persistence time of about $100 \mathrm{msec}$ but greater than a velocity-limited tracking time of about $18 \mathrm{msec}$ per deg of visual angle.
\end{abstract}

An illusion of continuous motion can occur when smooth pursuit movement of the eyes, which is normally elicited by a continuously moving stimulus, is elicited, instead, by a series of discrete spatially separated stimuli (Adler \& Grusser, 1979; Heywood, 1973; Lamontagne, 1973; Morgan \& Turnball, 1978). Using a type of display that allows us to infer continuous eye movements without directly measuring them, we report a parametric determination of the spatiotemporal conditions that give rise to this phenomenon, which we call "pursuit-locked apparent motion."

We presented a luminous vertical line in a sequence of discrete positions horizontally placed farther and farther to the right in equal steps, D, across the screen of a computer-controlled cathode ray tube (CRT). Following its presentation in the last position to the right, the line recycled, beginning again at the left. In each position, the luminous line was periodically displayed in brief pulses at a sufficient rate that the line, when stationary, appeared steady rather than flickering.

In accordance with Korte's third law of apparent motion (Korte, 1915; also see Farrell, 1983; Larsen, Farrell, \& Bundesen, 1983; Shepard \& Judd, 1976; Shepard \& Cooper, 1982, chap. 16; Shepard \& Zare, 1983), the standard illusion of apparent motion emerged when the distance, D, between successive positions was not too large relative to the time, T, or "stimulus onset asynchrony" (SOA) between those successive positions. The appearance, rather than being of each line going off and the next line coming on to its right, was then of a single

This work was supported by National Science Foundation Grants BNS 75-02806 and BNS 80-05517 to Stanford University and by National Institute of Mental Health Grant 5-T32MH14267 to New York University. In connection with the main experiment, the authors wish to thank George Sperling for the use of his experimental equipment and Joan Garey for her volunteer participation as the second observer. The authors' mailing address is: Department of Psychology, Stanford University, Stanford, CA 94305. line hopping through the intervening space between each position and the next.

For a particular range of values of $\mathrm{T}$ and $\mathrm{D}$, however, continued viewing spontaneously led to an entirely different and much more striking illusion. The experience of a single line jumping across the screen gave way to the appearance of a set of several closely spaced parallel vertical lines moving at a constant velocity across the entire screen. There was no longer any experience of particular positions at which the moving pattern "alighted" or varied in any way during what now appeared as a completely uniform rectilinear translation across the screen.

The explanation for this alteration in subjective experience was clear: The motion of the eyes, in tracking the successively rightward shifted line, changed from a series of discrete saccadic jumps to a constant-velocity glide across the entire screen. Consequently, the successive flashes of the line in each position no longer coincided on the retina, but each was laterally displaced relative to the preceding by a small constant amount, determined by the velocity of the eye movement (and, hence, by $\mathrm{D} / \mathrm{T}$ ).

This explanation, which has been called the "retinal painting hypothesis" (Morgan, 1981), has been supported by a large number of studies (Adler \& Grusser, 1979; Heywood, 1973; Lamontagne, 1973; Morgan \& Turnbull, 1978). For example, Adler and Grusser reported that when observers tracked a moving target across a background of flashing dots, the dots appeared to be organized into a pattern of vertical stripes. The investigators were able to predict the apparent separation of the illusory stripes by the product of the velocity of pursuit eye movements and the time interval separating successive flashes of the background dots. Their finding is consistent with the assumption that the transition in our experiment from apparent jerky motion of a single line to continuous motion of a set of parallel lines occurred when observers changed from saccadic to continuous tracking movements. 
If the eyes were to move at exactly the velocity $\mathrm{D} / \mathrm{T}$, a line flashed $\mathrm{n}$ times in each position would project $\mathrm{n}$ evenly spaced vertical lines on each retina, and the $n$ flashes of the next line to the right would exactly coincide on the retina with the last $\mathrm{n}$ flashes. Moreover, if the delay $\mathrm{T}$ were not too great, the new flashes would arrive while the retinal effects of the preceding flashes were still persisting. Under these conditions, the brain would have two pieces of information for constructing its interpretation of what is taking place on the external screen: First, it would have the information that a projected pattern of persisting parallel lines is fixed with respect to each retina; second, it would have the information that the eyes are moving from left to right at a constant velocity across the screen. The brain's most plausible inference would then be that a pattern of parallel lines is moving at a constant velocity across the screen.

We parametrically varied the distance, $\mathrm{D}$, and the time, $\mathrm{T}$, between successively displaced flashes in order to establish the spatiotemporal conditions for the occurrence of this phenomena of pursuit-locked apparent motion.

\section{METHOD}

\section{Subjects}

Two observers with corrected-to-normal vision (including one of the authors, J.E.F.) served as observers in the main experiment.

\section{Stimuli}

A computer presented a luminous vertical line segment in a succession of positions on an otherwise dark CRT screen with P4 phosphor, each position following the preceding by a fixed interval of time, $T$, displaced to the right by a fixed distance, $D$, of $0.72,1.08,1.44$, or $1.8 \mathrm{deg}$ of visual angle. The line was briefly flashed in each position $n$ times, where $n=D / 0.36$. The time between line presentations was $T / n$, ensuring that during smooth tracking movements, the lateral separation between the retinal images of successive flashes of a stationary line would be constant (at $0.36 \mathrm{deg}$ of visual angle). The vertical line segment subtended a visual angle of approximately $1.96 \mathrm{deg}$ and had an intensity of $151 \mathrm{~cd} / \mathrm{m}^{2}$. Observers viewed the CRT display in the dark while resting their heads inside a cushioned brace that prevented head movements.

\section{Procedure}

The experiment included three different stimulus conditions, as follows.

In Condition A, each trial was initialized with an SOA of $\mathrm{T}=180 \mathrm{msec}$, which was too long to yield pursuit-locked apparent motion. Observers then repeatedly decreased the time interval between successive displacements of the line, by pressing a left-hand button, which decreased $\mathrm{T}$ each time by oneeleventh of its previous value. Observers followed the horizontal displacement of the line with their eyes while decreasing $T$, until they saw a completely filled-in set of parallel lines moving continuously across the screen. When observers first achieved this illusion on a particular trial, they pressed a right-hand button, which recorded that critical T, referred to as SOA1.

In Condition B, each trial began with pursuit-locked apparent motion, and observers decreased $\mathrm{T}$ still further by pushing the left-hand button until the illusion of continuous motion of the line(s) broke down. They then pushed the right-hand button, recording that critical $\mathrm{T}$, referred to as SOA2.
In Condition C, observers began, as in Condition A, by decreasing $\mathrm{T}$ until they had achieved pursuit-locked apparent motion. By pressing another button, they then increased the distance, $D$, separating the successive positions of the target, in steps of $.36 \mathrm{deg}$ of visual angle, until the illusion of continuous motion disappeared.

Each condition was separated into 4 blocks of eight trials. Each angular separation, D, occurred twice within each block of trials. The order of presentations was randomized within each block, and the order of stimulus conditions was randomized across blocks. Observers completed 12 blocks of trials over the course of 3 consecutive days.

\section{RESULTS AND DISCUSSION}

Figure 1 shows the data obtained from the two observers in the main experiment. The mean critical $\mathrm{T}$ val-
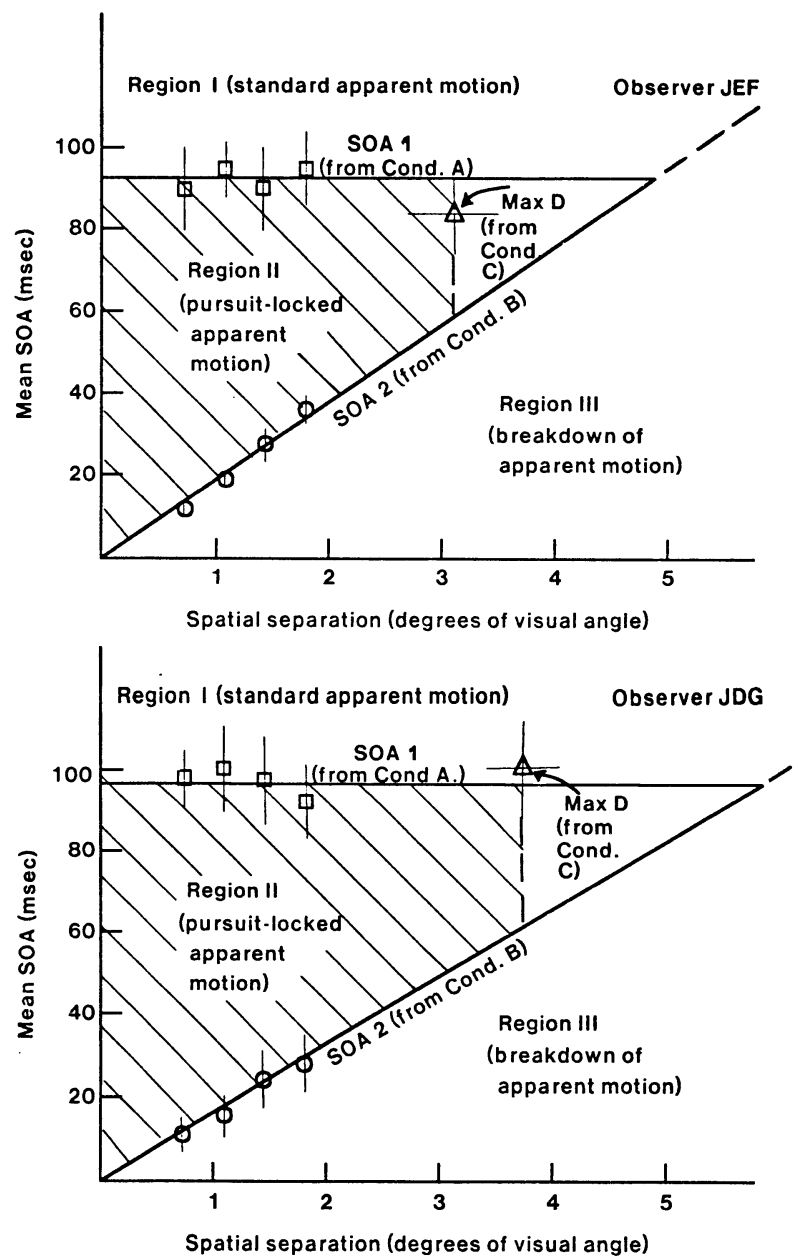

Figure 1. Data obtained from the two observers in the main experiment. For decreasing SOAs, squares indicate the mean $T$ values at which pursuit-locked apparent motion emerged (SOA1), and circles indicate the mean $T$ values at which the illusion broke down (SOA2). Triangles indicate the mean values for the maximum temporal and spatial separations ( $T$ and $D)$ for pursuit-locked apparent motion, as obtained in Condition $\mathrm{C}$. Each line segment through a plotted point represents plus and minus one standard deviation. 
ues that achieved the initial appearance (SOA1) and the subsequent disappearance (SOA2) of pursuit-locked apparent motion are plotted as a function of the spatial separation, D. Squares represent the mean value of SOA1, circles represent the mean value of SOA2, and triangles represent the mean value for the limiting temporal and spatial separations that were recorded in Condition $\mathrm{C}$. The line segments through each estimate represent plus and minus one standard deviation.

For both observers, the maximum temporal separation that yielded pursuit-locked apparent motion (SOA1) was constant across spatial separations. Evidently, observers can track an intermittently illuminated target with smooth pursuit eye movements when successive samples of the moving stimulus are separated by a time interval no longer than about $100 \mathrm{msec}$. This is in approximate agreement with previous findings that observers may initiate smooth pursuit eye movements when a moving target is presented every $150 \mathrm{msec}$ or less (Morgan \& Turnball, 1978; Westheimer, 1954). Presumably, the observed limit on the maximum temporal separation for pursuit-locked apparent motion depends on the retinal persistence of each sample of the moving stimulus.

Figure 1 also indicates that the minimum temporal separation for pursuit-locked apparent motion (SOA2) is proportional to the distance separating successive line positions. Evidently, pursuit-locked apparent motion is constrained by a limit on the velocity of pursuit eye movements. The slope of the linear function relating SOA2 to distance, D, is an estimate of the maximum velocity of pursuit eye movements and was approximately $51 \mathrm{deg} / \mathrm{sec}$ for Observer J.E.F. and $59 \mathrm{deg} / \mathrm{sec}$ for Observer J.D.G. These estimates are quite close to the estimate of about $60 \mathrm{deg} / \mathrm{sec}$ for the maximum velocity of smooth pursuit eye movements that Westheimer (1954) originally found for observers who were attempting to track an intermittently illuminated moving target. (For velocities greater than $60 \mathrm{deg} / \mathrm{sec}$, the eye movements of his observers tended to become saccadic.)

Finally, Figure 1 indicates that the maximum spatial separation inducing pursuit-locked apparent motion was approximately $3.1 \mathrm{deg}$ for Observer J.E.F. and $3.7 \mathrm{deg}$ for Observer J.D.G. Apparently, this limit is not a consequence of the velocity constraint on pursuit eye movements. If the maximum spatial separation were solely determined by the maximum velocity of pursuit-locked apparent motion, the maximum spatial separation should have been predictable from the product of the maximum temporal separation for pursuit eye movements (estimated in Condition A by SOA1) and the maximum velocity of pursuit eye movements (estimated in Condition B by D/SOA2). The predicted spatial limits (4.8 and $5.7 \mathrm{deg}$. for Observers J.E.F. and J.D.G., respectively) are greater than the observed spatial limits (3.1 and $3.7 \mathrm{deg}$ ), indicating that, in Condition C, pursuit-locked apparent motion broke down at velocities that were below the velocity limit on pursuit eye movements.

We also estimated this spatial limit in five observers who participated in a preceding pilot study. As in the main experiment, these observers first decreased $\mathrm{T}$ until they achieved pursuit-locked apparent motion, and then increased D until the illusion broke down. However, the experiment used in the pilot study did not permit control of the rate at which the screen was refreshed, and, hence, the retinal spacing between images of successive flashes of the vertical line increased with the velocity of eye movements. Nevertheless, we obtained spatial limits on the maintenance of pursuit-locked apparent motion of 2.4, 2.9, 3.0, 3.1, and 3.1 deg for Observers S.L.Z., T.H.P., R.N.S., E.J.K., and J.E.F., respectively. Thus, the estimate of the spatial limit for J.E.F. was the same in that pilot experiment as in the ensuing main experiment (viz., 3.1 deg in both experiments).

This spatial constraint on pursuit eye movements is consistent, also, with one determined by Kommerell and Klein (1971). They reported that a stabilized retinal afterimage can elicit smooth pursuit eye movements if located no more than 2 to $3 \mathrm{deg}$ from the fovea centralis. Since the afterimage was stabilized with respect to the retina, when their observers tried to fixate the image, the target appeared to move in the direction of their eye movements. When the distance between the afterimage and the fovea centralis was greater than 3 to $4 \mathrm{deg}$, smooth eye movement was interrupted by saccades.

We can summarize all of our results in terms of the three regions, I, II, and III, indicated in Figure 1. For a single line (1) that was periodically illuminated in brief pulses beyond the rate required for flicker fusion when the line is stationary and (2) that was discontinuously displaced a distance $\mathrm{D}$ to the right every $\mathrm{T}$ msec, we found three distinct phenomena depending on the values of $\mathrm{D}$ and $\mathrm{T}$. For $\mathrm{T}>(18 \mathrm{msec} / \mathrm{deg}) \mathrm{D}$, and in accordance with Korte's third law, we obtained some variety of apparent motion. For $T>100 \mathrm{msec}$, this was standard apparent motion, in which the single line appeared to jump from each displayed position to the next (Region I). For $\mathrm{T}<100 \mathrm{msec}$ and $\mathrm{D}<$ about $3 \mathrm{deg}$, however, we obtained pursuit-locked apparent motion in which a bundle of $\mathrm{n}$ closely spaced parallel lines (corresponding to the $\mathrm{n}$ flashes of the line in each position) appeared to move with constant velocity across the entire display (Region II). Finally, for $\mathrm{T}<(18 \mathrm{msec} / \mathrm{deg}) \mathrm{D}$, apparent motion of the line as such broke down (Region III). Within this last region, the observers experienced more than one position of the line as illuminated at once, but without any motion of the positions themselves. Some described the appearance as resembling a spatially extended light moving to the right behind a fixed "fence" with several adjacent narrow vertical spaces (corresponding to the fixed positions of the lines) between the "boards" being illuminated at any moment. The experienced motion was thus of this diffuse "illumination" and not of the spatially localized lines. 
As a final, methodological comment, from the particular nature of the visual experience that our pulsating display elicited within Region II, we are able to infer the exact form and velocity of pursuit eye movements in the absence of a moving stimulus, without requiring any direct measurements of those eye movements.

\section{REFERENCES}

Adler, B., \& Grusser, O. J. (1979). Apparent movement and appearance of periodic strips during eye movements across a stroboscopically illuminated random dot pattern. Experimental Brain Research, 37, 537-550.

Boring, E. G. (1942). Sensation and perception in the history of experimental psychology. New York: Appleton-Century-Crofts.

FARrell, J. E. (1983). Visual transformations underlying apparent movement. Perception \& Psychophysics, 33, 85-92.

HeYwood, S. (1973). Pursuing stationary dots: Smooth eye movements and apparent movement. Perception, 2, 181-195.

Kommerell, G., \& KLein, U. (1971). Über die Visuelle Regelung der Okulomotorik. Vision Research, 11, 905-920.

KorTE, A. (1915). Kinematoskopische Untersuchungen. Zeitschrift für Psychologie, 72, 193-296.
Lamontagne, C. (1973). A new experimental paradigm for the investigation of the secondary system of human visual motion perception. Perception, 2, 167-180.

Larsen, A., Farrell, J. E., \& Bundesen, C. (1983). Short- and long-range processes in visual apparent motion. Psychological Research, 45, 11-18.

Morgan, M. J. (1981). How pursuit eye movements can convert temporal into spatial information. In D. F. Fisher, R. A. Monty, \& J. W. Senders (Eds.), Eye movements: Cognition and visual perception (pp. 111-133). Hillsdale, NJ: Erlbaum.

Morgan, M. J., \& Turnbull, D. F. (1978). Smooth eye tracking and the perception of motion in the absence of real movement. Vision Research, 18, 1053-1059.

Shepard, R. N., \& CoOper, L. A. (1982). Mental images and their transformations. Cambridge, MA: MIT Press/Bradford Books.

She Pard, R. N., \& Judd, S. A. (1976). Perceptual illusion of rotation of three-dimensional objects. Science, 191, 952-954.

ShePARD, R. N., \& ZARE, S. (1983). Path-guided apparent motion. Science, 220, 632-634.

Westheimer, G. (1954). Eye movement responses to a horizontally moving stimulus. Archives of Ophthalmology, 52, 932-941.

(Manuscript received for publication February 13, 1984.) 